\title{
A Numerical Solution of Schrödinger's Equation in the Continuum ${ }^{1}$
}

\author{
W. Futterman, E. Osborne, and David S. Saxon ${ }^{2}$
}

\begin{abstract}
Continuum solutions of Schrödinger's equation for two particles that interact according to a central Yukawa potential are obtained by numerical integration. The resulting wave functions are tabulated, as are the phase shifts which determine the asymptotic behavior of the solutions.
\end{abstract}

\section{Introduction}

The solution of the Schrödinger equation for two particles that interact according to the Yukawa potential,

$$
V(r)=C \frac{e^{-r / r_{0}}}{r / r_{0}}
$$

is unknown in closed form. Numerical solutions of this problem are of some interest from a purely formal as well as physical viewpoint, since the literature of recent years shows increased activity in the use of a variational principle ${ }^{3,4}$ to obtain certain parameters of physical consequence. As yet, however, with respect at least to solutions in the continuum there exist no intrinsic criteria for the deviation of such approximate results from the correct values. An exact solution of the problem, of course, permits an exact calculation of these parameters, thus providing a comparison by which the validity and usefulness of the variational principle can be gaged. ${ }^{5}$ Further, although the Yukawa interaction (1) is an idealized one, it nonetheless forms a reasonable basis for the description of physical phenomena such as the photoelectric disintegration of the deuteron and the so-called deuteron-stripping process. ${ }^{6}$

\section{Schrödinger Equation}

For the Yukawa interaction (1) the Schrödinger equation, expressed in the relative coordinates of the two particles, is

$$
\left\{\nabla^{2}+\frac{2 m}{\hbar^{2}}[E-V(r)]\right\} \Psi(\boldsymbol{r})=0,
$$

where $E$ is the energy of the system, and $m$ is the reduced mass. In virtue of the spherical symmetry of $V(r)$, the solution of (2) can be decomposed into spherical harmonics; that is, $\Psi(\boldsymbol{r})$ can be written in the form

$$
\Psi(\boldsymbol{r})=\sum_{l, m} a_{l, m} \Psi_{l}(r) Y_{l}^{m}(\theta, \phi),
$$

1 The preparation of this paper was sponsored (in part) by the Office of Naval Research, USN

2 National Bureau of Standards, Los Angeles, Calif.

3 Lippman and Schwinger, Phys. Rev. 79, 469 (1950).

4 Blatt and Jackson, Phys. Rev. 76, 18 (1949).

${ }_{5}^{5}$ In particular, reference is made to a calculation of differential cross sections, ${ }^{5}$ In particular, reference is made to a calculation
using variational methods, which is in preparation.

using variational methods, which is in preparation.
6 Schiff, Phys. Rev. 78, 733 (1950); Serber, Phys. Rev. 72, 1008 (1947). (Applications of our results to these problems are in preparation.) where the $a_{l, m}$ are arbitrary constants, and the $Y_{l}^{m}$ are the usual normalized spherical harmonics. The radial functions $\Psi_{l}(r)$ then satisfy the equation

$$
\frac{1}{r^{2}} \frac{d}{d r}\left(r^{2} \frac{d \Psi_{l}}{d r}\right)+\left\{\frac{2 m}{\hbar^{2}}(E-V(r))-\frac{l(l+1)}{r^{2}}\right\} \Psi_{l}=0 .
$$

Introducing the function

$$
u_{l}(r)=r \Psi_{l}(r)
$$

and using (1), it is convenient to rewrite (4) in the dimensionless form

$$
\frac{d^{2} u_{l}}{d x^{2}}+\left\{K^{2}+\beta \frac{e^{-x}}{x}-\frac{l(l+1)}{x^{2}}\right\} u_{l}=0
$$

where

$$
\begin{aligned}
K^{2} & =2 m r_{0}^{2} E / \hbar^{2} \\
\beta & =2 m r_{0}^{2} C / \hbar^{2} \\
x & =r / r_{0} .
\end{aligned}
$$

and where the parameters $r_{0}$ and $C$ (or $\beta$ ) will be chosen to fit the low energy triplet neutron-proton data.

As a consequence of $(5), u_{l}(r)$ satisfies the boundary condition $u_{l}(0)=0$, and thus we seek the solution of (6) regular at $x=0$. It is easily established that in the neighborhood of the origin, this solution behaves as

$$
u_{l}(x) \sim x^{l+1}, \quad x \rightarrow 0 .
$$

It should be noted that the complete solution of the Schrödinger equation consists of a sum over all spherical harmonics. However, for sufficiently large $l$ the effect of the interaction term can be shown to be very small, and the solutions may be obtained by perturbation methods, such as the Born approximation. Consequently, the numerical integration of (6) need be carried out only for the first few $l$ values.

One should also remark that for sufficiently large values of $x$, no matter what value $l$ has, the interaction term becomes negligible, and hence the asymptotic form of $u_{l}$ must be

$$
u_{l}(x) \simeq x B_{l} \cos \delta_{l}\left[j_{l}(K x)-\tan \delta_{l} y_{l}(K x)\right],
$$


where $j_{l}(z)$ is the regular and $y_{l}(z)$ the irregular spherical Bessel function, ${ }^{7}$ of order $l$, and where the factor $\cos \delta_{l}$ is introduced for reasons of convenience. Thus the numerical integration need extend only to such values of $x$ that (11) is valid, as discussed in section 3 .

The physical significance of the phase shifts $\delta_{l}$ defined by (11) may be seen from the fact that in the absence of interaction, (11) holds exactly everywhere. In this special case, the requirement of regularity at the origin is satisfied only if each $\delta_{l}$ is zero. Thus the phase shifts measure the influence of the interaction on the asymptotic form of the field and so directly determine the scattering. ${ }^{8}$

\section{Procedure}

Integration of (6) is based upon the GaussJackson or $\Sigma^{2}$ difference method for reasons of accuracy and convenience. ${ }^{9}$ Let us rewrite (6) in the form

$$
\frac{d^{2} u}{d x^{2}}=g_{l}(x) u
$$

where

$$
g_{l}(x)=-\left(K^{2}+\beta \frac{e^{-x}}{x}-\frac{l(l+1)}{x^{2}}\right)
$$

In central difference notation

$$
u^{(r)}=\frac{1}{4 S}\left[\delta^{-2} F_{r}+\frac{1}{12} F_{r}-\frac{1}{240} \delta^{2} F_{r}+\ldots\right],
$$

where we tabulate

$$
F_{r}=4 S h^{2} g(x) u^{(r)},
$$

and where $h$ is the interval, $S$ is an arbitrary roundingoff factor, and $u_{l}^{(0)}=u_{l}(0)$.

Because (12) has a regular singular point at $x=0$ care must be taken to determine the $F_{0}$ for the various $l$ values. However, they are readily obtained from (10), which gives

$$
\begin{array}{ll}
l=0 & g_{0}(0) u_{0}^{(0)}=\text { constant } \\
l=1 & g_{1}(0) u_{1}^{(0)}=\text { constant } \\
l \geq 2 & g_{1}(0) u_{1}^{(0)}=0
\end{array}
$$

where the constants determine the otherwise arbitrary normalization.

The series (14) is approximated to the order of the second difference term, using the third difference as a predictor. As a consequence, $F_{1}$ and $F_{2}$ are needed as starting values in addition to $F_{0}$. These are obtained from (10), which is a suitable first approxima-

7 These functions are defined in P. M. Morse, Vibrations and sound, p. 316-317, and they are tabulated in "Tables of spherical Bessel function" (Columbia University Press, 1947)

8 Mott and Massey, The theory of atomic collisons (Oxford, 1949).

9 Samuel Herrick, Mathematical tables and other aids to computation 5, 61-67 (1951) (1951): (See also, page 131 of reference given in footnote 8.) Recently, P. O lowdin and A. S. Jolander, Arkiv für Fysik, Band 3, No. 11, (1951), claim an improvement over $\Sigma^{2}$ procedure on the basis that no initial Taylor expansion is needed. However, in our case no special difficulties were encountered in this respect. tion, as indicated by the rapid convergence of $F_{2}$ to a constant value. Three iterations were made at each point to assure the constancy of the tabulated value.

Keeping the interval small, $h=.05$, in this region of rapidly changing ordinate, we march out to $x=1$ where the interval is shifted to $h=.1$. The integration is then carried out to a large value of $x(\sim 5)$. Rather than joining this solution to (11), which is obtained by neglecting the interaction term entirely, a more refined procedure was used in which the interaction was treated as a small perturbation. By a modified WKB technique, ${ }^{10}$ the asymptotic solution can be obtained in the form

$$
u_{l}(x)=A_{l} \cos \delta_{l} \frac{z(x)}{\sqrt{z^{\prime}(x)}}\left[j_{l}(z(x))-\tan \delta_{l} y_{l}(z(x))\right]
$$

where the prime denotes differentiation with respect to $x$, and where $z$ is given by the semiconvergent series

$$
\begin{aligned}
z(x)=K x+ & \frac{1}{\left(1+1 / 4 K^{2}\right)} \beta \frac{e^{-x}}{x}[-1 \\
& \left.+\frac{1}{x}\left(\frac{1+3 / 4 K^{2}}{1+1 / 4 K^{2}}\right)-\cdots\right]+O\left(\frac{e^{-2 x}}{x^{2}}\right) .
\end{aligned}
$$

It is observed that as $x$ approaches infinity, $z(x)$ properly approaches $K x$. A few terms of this alternating series were always sufficient to give $z$ with satisfactory accuracy. The logarithmic derivative of (17) was equated to the numerically obtained logarithmic derivative of the numerical solution at its end point, thus determining the phase shift. The amplitude factor was then found by equating (17) to the numerical solutions.

In carrying out the actual computation, the original data was programmed to six significant figures on the IBM card-programmed calculator, so that at least four figures could be assured for the phase shifts. With a few exceptions, integrations were carried out to $x=5$ at all energies. An internal check on the error was maintained through the third differences, which were kept uniform and small.

To check the machine error a comparison of two integrations for $K^{2}=1, \beta=0$ was made with the known solutions $j_{l}(K x)$. For the cases $l=2$ with the end point $x=4$, and $l=3$ with $x=5$ there was, at most, a 0.0002 percent error in the numerical solution.

\section{Numerical Results}

As mentioned earlier, the constants in the Yukawa interaction were chosen to fit the low-energy triplet neutron-proton interaction. Using the tables of Blatt and Jackson, ${ }^{11}$ we choose

$$
\beta=2.36500, \quad r_{0}=1.35000 \times 10^{-13} \mathrm{~cm} .
$$

1(The details are given in the appendix.

11 See footnote 4. 
The parameter $K^{2}$ is easily expressed in terms of the incident neutron energy, ${ }^{12}$

$$
K^{2}=2.19700 \times 10^{-2} \mathrm{E}_{\text {lab. }} \text { (Mev). }
$$

Computations were carried out for the values of the energy, with the corresponding values of $K^{2}$ listed in table 1 .

TABLE 1

\begin{tabular}{|c|c|c|c|c|c|}
\hline $\begin{array}{c}E_{\text {lab. }}(\mathrm{Mev}) \ldots- \\
K^{2} \ldots \ldots\end{array}$ & 20 & 50 & 90 & 120 & 150 \\
\hline
\end{tabular}

The radial wave functions $u_{l}(x)$ corresponding to these parameters are tabulated in table 2 for $l=0(1) 5$ and for $x=0(0.1) 5$ (in a few cases for $x=0(0.1) 4.0$ or 4.2$)$. For values of $x$ that lie beyond the tabulated values, $u_{l}$ is given by (17) where the parameters $\delta_{l}$ are listed in table 3 . For convenience in physical applications, these functions have been normalized according to the rule

$$
\int_{0}^{\infty} u_{l}^{K}(x) u_{l}^{K^{\prime}}(x) d x=\frac{\pi}{2 K} \delta\left(K-K^{\prime}\right),
$$

where $u_{l}^{K}(x)$ is the solution when the energy corresponds to $\mathrm{K}$ and where $\delta\left(K-K^{\prime}\right)$ is the Dirac $\delta$-function. With this normalization the factors $A_{l}$, which appear in (17), are then simply unity.

Finally, for values of $l$ that lie beyond the tabulated values, the wave functions can be obtained by perturbation methods, such as the Born approximation, since, as already mentioned, the effects of the interaction are then very small.

\section{Appendix}

We seek asymptotic solutions of eq (6) valid when $x$ is so large that the term $\beta e^{-x} / x$ can be treated as a small pertubation. For this purpose we consider the general spherical Bessel function $c_{l}(z)$, which is defined as the general solution of the equation

$$
\frac{d^{2} c_{l}}{d z^{2}}+\frac{2}{z} \frac{d c_{l}}{d z}+\left[1-\frac{l(l+1)}{z^{2}}\right] c_{l}(z)=0,
$$

and which is thus expressible in the form

$$
c_{l}(z)=\alpha \jmath_{l}(z)+\beta y_{l}(z) .
$$

Now as $x$ increases, $u_{l}(x)$ is ultimately given by

$$
u_{l}(x) \sim K x c_{l}(K x),
$$

as indicated in eq (11). Hence, we try to find an asymptotic solution of $(6)$ of the form

$$
u_{l}(x)=A(x) z(x) c_{l}[z(x)] .
$$

12 For convenience in physical applications, $K^{2}$ is expressed in terms of the energy in the laboratory system of coordinates in which the proton is initially at energy in the laboratory system of coordinates in which the proton is initially at
rest. In (2) the energy that appears is that in the coordinate system in which rest. In $(2)$ the energy that
the center of mass is at rest.
Substitution into (6) and use of $(A-1)$ then yields

$$
\begin{aligned}
& {\left[2 A^{\prime} z z^{\prime}+A z z^{\prime \prime}\right] \frac{d c_{l}}{d z}-A z z^{\prime 2}\left[1-\frac{l(l+1)}{z^{2}}\right] c_{l}(z)} \\
& +\left\{A z\left[K^{2}+\frac{\beta e^{-x}}{x}-\frac{l(l+1)}{x^{2}}\right]+2 A^{\prime} z^{\prime}+A z^{\prime \prime}+A^{\prime \prime} z\right\} c_{l}(z)=0,
\end{aligned}
$$

where the prime denotes differentiation with respect to $x$. The unknown functions $A(x)$ and $z(x)$ are now determined by setting the coefficients of $c_{l}(z)$ and $d c_{l} / d z$ separately equal to zero. The latter gives at once

$$
A^{2} z^{\prime}=\text { constant }
$$

whence, eliminating $A$, the former gives

$$
\begin{aligned}
-z^{\prime 2}\left[1-\frac{l(l+1)}{z^{2}}\right]+K^{2}[ & \left.1-\frac{l(l+1)}{K^{2} x^{2}}\right] \\
& +\frac{\beta e^{-x}}{x}+\sqrt{z^{\prime}} \frac{d^{2}}{d x^{2}}\left(\frac{1}{\sqrt{z^{\prime}}}\right)=0,
\end{aligned}
$$

which is an exact equation for $z(x)$. Note that for $\beta=0$, $z=K x$ is properly a solution of this equation. Adopting $\beta$ as a convenient parameter of smallness (since it occurs multiplied by the small factor $e^{-x} / x$ ), we now find approximate solutions by expanding $z(x)$ in a power series in $\beta$ as follows:

$$
z(x)=K x+\underset{1}{\infty} \beta^{n} v_{n}(x),
$$

whence, by substitution into (A-6) we obtain upon setting the coefficients of like powers of $\beta$ equal to zero

$$
\frac{v_{1}^{\prime \prime \prime}}{4 K^{2}}+\left[1-\frac{l(l+1)}{K^{2} x^{2}}\right] v_{1}^{\prime}+\frac{l(l+1)}{K^{2} x^{3}} \tilde{v}_{1}=\frac{e^{-x}}{2 K x}
$$

and similarly for $v_{2}$, etc. Since $x$ is presumed large, we solve (A-8) approximately by writing

$$
v_{1}=\frac{e^{-x}}{2 K} \sum_{n=0} \frac{a_{n}}{x^{n+1}}
$$

which finally yields

$$
\begin{aligned}
& a_{0}=-\frac{1}{1+1 / 4 K^{2}} \\
& a_{1}=-\frac{1+3 / 4 K^{2}}{1+1 / 4 K^{2}} a_{0} \\
& a_{2}=-\frac{1}{1+4 K^{2}}\left\{[6-4 l(l+1)] a_{0}+2\left(4 K^{2}+3\right) a_{1}\right\}
\end{aligned}
$$

Assembling these results, we then obtain eq (17) and (18). 
TABLe 2

\begin{tabular}{|c|c|c|c|c|c|c|c|c|c|c|c|c|c|}
\hline \multicolumn{7}{|c|}{$\mathrm{E}_{1 \mathrm{ab} .}=20 \mathrm{Mev}$} & \multicolumn{7}{|c|}{$\mathrm{E}_{1 \mathrm{ab} .}=50 \mathrm{Mev}$} \\
\hline$x$ & $u_{0}(x)$ & $u_{1}(x)$ & $u_{2}(x)$ & $u_{3}(x)$ & $u_{4}(x)$ & $u_{5}(x)$ & $x$ & $u_{0}(x)$ & $u_{1}(x)$ & $u_{2}(x)$ & $u_{3}(x)$ & $u_{4}(x)$ & $u_{5}(x)$ \\
\hline 0.1 & 0.2592 & 0.0042 & & & & & 0.1 & 0.24687 & 0.0076 & 0.0001 & & & \\
\hline .2 & .4592 & .0158 & 0.0003 & & & & .2 & .43753 & .0286 & .0009 & & & \\
\hline .3 & .6128 & .0338 & .0010 & 0.00002 & & & .3 & .58090 & .0610 & .0031 & 0.0001 & & \\
\hline .4 & .7297 & .0572 & .0022 & .00007 & & & .4 & .68385 & .1026 & .0070 & .0004 & 0.00002 & \\
\hline .5 & .8134 & .0852 & .0042 & .00018 & 0.00001 & & .5 & .75211 & .1519 & .0132 & .0009 & .00005 & \\
\hline .6 & .8701 & .1172 & .0071 & .00036 & .00002 & 0.000001 & .6 & .79046 & .2073 & .0221 & .0018 & .00012 & 0.00001 \\
\hline .7 & .9041 & .1526 & .0110 & .00065 & .00003 & .000001 & .7 & .80298 & .2675 & .0339 & .0032 & .00025 & .00002 \\
\hline .8 & .9189 & .1911 & .0160 & .00109 & .00006 & .000003 & .8 & .79317 & .3312 & .0489 & .0054 & .00048 & .00004 \\
\hline .9 & .9174 & .2322 & .0222 & .00172 & .00011 & .000006 & .9 & .76407 & .3972 & .0673 & .0084 & .00084 & .00007 \\
\hline 1.0 & .9021 & .2755 & .0297 & .00257 & .00018 & .000011 & .10 & .71839 & .4644 & .0892 & .0126 & .00140 & .00013 \\
\hline 1.1 & .8749 & .3207 & .0386 & .00370 & .00029 & .000019 & 1.1 & .65855 & .5318 & .1147 & .0179 & .00221 & .00023 \\
\hline 1.2 & .8375 & .3675 & .0490 & .00515 & .00044 & .000031 & 1.2 & .58674 & .5983 & .1438 & .0247 & .00335 & .00038 \\
\hline 1.3 & .7915 & .4157 & .0609 & .00698 & .00065 & .000050 & 1.3 & .50498 & .6630 & .1765 & .0332 & .00489 & .00060 \\
\hline 1.4 & .7381 & .4648 & .0744 & .00925 & .00093 & .000078 & 1.4 & .41517 & .7250 & .2126 & .0435 & .00694 & .00093 \\
\hline 1.5 & .6784 & .5147 & .0895 & .01199 & .00130 & .000116 & 1.5 & .31905 & .7833 & .2520 & .0558 & .00959 & .00138 \\
\hline 1.6 & .6133 & .5651 & .1063 & .01529 & .00177 & .000169 & 1.6 & .21830 & .8373 & .2944 & .0702 & .01295 & .00199 \\
\hline 1.7 & .5436 & .6158 & .1249 & 01918 & .00237 & .000241 & 1.7 & .11449 & .8862 & .3396 & .0870 & .01714 & .00281 \\
\hline 1.8 & .4702 & .6664 & .1451 & .02374 & .00312 & .000336 & 1.8 & .00912 & .9291 & .3873 & .1062 & .02229 & .00388 \\
\hline 1.9 & .3937 & .7168 & .1671 & .02903 & .00404 & .000461 & 1.9 & -.09637 & .9656 & .4371 & .1279 & .02852 & .00527 \\
\hline 2.0 & .3147 & .7667 & .1908 & .02509 & .00516 & .000620 & 2.0 & -.20062 & .9951 & .4885 & .1522 & .03597 & .00702 \\
\hline 2.1 & .2338 & .8158 & .2162 & .04200 & .00650 & .000823 & 2.1 & -.30236 & 1.0170 & .5410 & .1792 & .04476 & .00921 \\
\hline 2.2 & .1515 & .8639 & .2434 & .04981 & .00811 & .001077 & 2.2 & -.40037 & 1. 0309 & .5943 & .2089 & .05503 & .01192 \\
\hline 2.3 & .0685 & .9108 & .2721 & .05858 & .01000 & .001392 & 2.3 & -.49350 & 1. 0365 & .6476 & .2411 & .06690 & .01522 \\
\hline 2.4 & -.0150 & .9561 & .3025 & .06835 & .01222 & .001778 & 2.4 & -.58071 & 1. 0336 & .7005 & .2760 & .08049 & .01920 \\
\hline 2.5 & -.0984 & .9997 & .3345 & .07919 & .01479 & .002248 & 2.5 & -.66103 & 1.0220 & .7524 & .3133 & .09592 & .02395 \\
\hline 2.6 & -.1813 & 1. 0413 & .3679 & .09114 & 01777 & .002814 & 2.6 & -.73357 & 1. 0017 & .8027 & .3529 & .11330 & .02957 \\
\hline 2.7 & -.2632 & 1. 0808 & .4027 & .10425 & .02117 & .003491 & 2.7 & -.79757 & 0.9727 & .8507 & .3947 & .13270 & .03616 \\
\hline 2.8 & -.3439 & 1. 1178 & .4388 & .11855 & .02506 & .004294 & 2.8 & -.85234 & .9350 & .8959 & .4384 & .15420 & .04382 \\
\hline 2.9 & -.4228 & 1. 1522 & .4761 & .13408 & .02946 & .005240 & 2.9 & -.89732 & .8891 & .9376 & .4837 & .11786 & .05265 \\
\hline 3.0 & -.4997 & 1. 1837 & .5146 & 15088 & .03442 & .006349 & 3.0 & -.93204 & .8350 & .9753 & .5305 & .20272 & .06275 \\
\hline 3.1 & -.5742 & 1. 2122 & .5539 & .16896 & .03998 & .007638 & 3.1 & -.95617 & .7733 & 1.0084 & .5782 & .23178 & .07424 \\
\hline 3.2 & -.6460 & 1. 2375 & .5941 & 18835 & .04618 & .009130 & 3.2 & -.96946 & .7045 & 1. 0363 & .6266 & .26204 & .08720 \\
\hline 3.3 & -.7148 & 1. 2593 & .6250 & .20907 & .05306 & .010846 & 3.3 & -.97182 & .6291 & 1.0587 & .6753 & .29445 & .10173 \\
\hline 3.4 & -.7802 & 1. 2777 & .6765 & .23111 & .06068 & .012811 & 3.4 & -.96326 & .5478 & 1. 0749 & .7239 & .32895 & .11791 \\
\hline 3.5 & -.8421 & 1.2923 & .7183 & .25449 & .06906 & .015049 & 3.5 & -.94389 & .4613 & 1.0847 & .7718 & .36546 & .13583 \\
\hline 3.6 & -.9000 & 1. 2031 & .7603 & .27918 & .07825 & .017587 & 3.6 & -.91397 & .3704 & 1.0877 & .8186 & .40383 & .15556 \\
\hline 3.7 & -.9539 & 1. 3099 & .8024 & .30518 & .08829 & .020451 & 3.7 & -.87384 & .2759 & 1.0835 & .8639 & .44392 & .17715 \\
\hline 3.8 & -1.0034 & 1. 3127 & .8443 & .33247 & .09922 & .023671 & 3.8 & -.82399 & .1787 & 1. 0721 & .9071 & .48555 & .20064 \\
\hline 3.9 & -1.0483 & 1. 3114 & .8860 & .36101 & .11107 & .027275 & 3.9 & -.76497 & .0798 & 1.0531 & .9478 & .52850 & .22607 \\
\hline 4.0 & -1.0885 & 1. 3058 & .9271 & .39077 & .12388 & .031293 & 4.0 & -.69746 & -.0198 & 1.0266 & .9854 & .57262 & .25345 \\
\hline 4.1 & -1.1238 & 1. 2961 & .9675 & .42169 & .13768 & .025758 & 4.1 & -.62222 & -.1193 & 0.9926 & 1.0194 & -... & .28276 \\
\hline 4.2 & -1.1541 & 1. 2820 & 1.0070 & .45374 & .15251 & .040700 & 4.2 & -.54008 & -.2176 & .9511 & 1.0495 & - & .31398 \\
\hline 4.3 & -1.1792 & 1. 2636 & 1.0454 & - & .16837 & .046152 & 4.3 & -.45197 & -.3137 & .9024 & 1.0750 & -...... & .34706 \\
\hline 4.4 & -1.1990 & 1. 2410 & 1.0826 & -..... & .18531 & .052147 & 4.4 & -.35887 & -.4067 & .8466 & 1.0957 & - & .38194 \\
\hline 4.5 & -1.2135 & 1. 2141 & 1. 1182 & -..... & .20333 & .058717 & 4.5 & -.26180 & -.4957 & .7840 & 1.1110 & ......... & .41850 \\
\hline 4.6 & -1.2226 & 1. 1830 & 1. 1522 & & .22245 & .065895 & 4. 6 & -.16184 & -.5796 & .7151 & 1. 1206 & - & .45664 \\
\hline 4.7 & -1.2262 & 1. 1478 & 1. 1844 & -...... & .24269 & .073714 & 4.7 & -.06009 & -.6577 & .6404 & 1. 1243 & -.... & .49621 \\
\hline 4.8 & -1.2244 & 1. 1085 & 1. 2145 & ....... & .26405 & - & 4.8 & .04232 & -.7291 & .5603 & 1.1216 & -...... & .53704 \\
\hline 4.9 & -1.2172 & 1. 0652 & 1. 2424 & -....... & .28652 & - & 4.9 & .14426 & -.7932 & .4755 & 1. 1124 & -...... & .57894 \\
\hline 5.0 & -1.2045 & 1.0182 & 1. 2679 & $\ldots$ & .31011 & - & 5.0 & .24461 & -.8491 & .3867 & 1.0965 & ....... & .62169 \\
\hline & & & & & & & 5.1 & .34227 & -.8964 & .2946 & 1. 0738 & - & .66504 \\
\hline
\end{tabular}


Table 2.-Continued

\begin{tabular}{|c|c|c|c|c|c|c|c|c|c|c|c|c|c|}
\hline \multicolumn{7}{|c|}{$\mathrm{E}_{\mathrm{lab} \cdot}=90 \mathrm{Mev}$} & \multicolumn{7}{|c|}{$\mathrm{E}_{1 \mathrm{ab} \cdot}=120 \mathrm{MeV}$} \\
\hline$x$ & $u_{0}(x)$ & $u_{1}(x)$ & $u_{2}(x)$ & $u_{3}(x)$ & $u_{4}(x)$ & $u_{5}(x)$ & $x$ & $u_{0}(x)$ & $u_{1}(x)$ & $u_{2}(x)$ & $u_{3}(x)$ & $u_{4}(x)$ & $u_{5}(x)$ \\
\hline 0.1 & 0.24151 & 0.01070 & 0.00025 & & & & 0.1 & 0.24053 & 0.01259 & 0.00035 & & & \\
\hline .2 & .42629 & .04031 & .00192 & 0.00007 & & & .2 & .42279 & .04734 & .00270 & 0.00011 & & \\
\hline .3 & .56129 & .08540 & .00622 & .00034 & 0.00001 & & .3 & .55291 & .09995 & .00873 & .00056 & 0.00003 & \\
\hline .4 & .65235 & .14282 & .01417 & .00104 & .00006 & & .4 & .63614 & .16631 & .01980 & .00170 & .00012 & 0.00001 \\
\hline .5 & .70460 & .20961 & .02658 & .00246 & .00018 & & .5 & .67735 & .24250 & .03698 & .00402 & .00035 & .00002 \\
\hline .6 & .72266 & .28303 & .04406 & .00494 & .00044 & & .6 & .68126 & .32475 & .06096 & .00804 & .00084 & .00007 \\
\hline .7 & .71078 & .36046 & .05704 & .00887 & .00093 & 0.0001 & .7 & .65246 & .40946 & .09215 & .01436 & .00175 & .00018 \\
\hline .8 & .67289 & .43943 & .09577 & .01465 & .00177 & .0002 & .8 & .59550 & .49324 & .13062 & .02358 & .00332 & .00038 \\
\hline .9 & .61274 & .51762 & .13029 & .02270 & .00310 & .0004 & .9 & .51493 & .57288 & .17613 & .03628 & .00579 & .00076 \\
\hline 1.0 & .53391 & .59283 & .17048 & .03341 & .00511 & .0006 & 1. 0 & .41527 & .64547 & .22814 & .05300 & .00948 & .00139 \\
\hline 1.1 & .43986 & .66303 & .21603 & .04717 & .00800 & .0011 & 1.1 & .30104 & .70835 & .28581 & .07421 & .01473 & .00239 \\
\hline 1. 2 & .33396 & .72634 & .26646 & .06434 & .01200 & .0018 & 1. 2 & .17670 & .75922 & .34805 & .10029 & .02193 & .00390 \\
\hline 1.3 & .21946 & .78108 & .32112 & .08522 & .01735 & .0029 & 1.3 & .04665 & .79614 & .41352 & .13147 & 03146 & .00611 \\
\hline 1.4 & .09952 & .82575 & .37923 & .11002 & .02434 & .0044 & 1. 4 & -.08488 & .81757 & .48071 & .16785 & 04373 & .00920 \\
\hline 1.5 & -.02281 & .85910 & .43986 & .13892 & .03322 & .0064 & 1.5 & -.21384 & .82240 & .54793 & .20937 & .05913 & .01343 \\
\hline 1.6 & -.14462 & .88008 & .50196 & .17199 & .04428 & .0092 & 1.6 & -.33643 & .81001 & .61337 & .25577 & .07801 & .01905 \\
\hline 1.7 & -.26316 & .88793 & .56439 & .20919 & .05779 & .0129 & 1. 7 & -.44918 & .78020 & .67517 & .30665 & 10069 & .09635 \\
\hline 1.8 & -.37584 & .88212 & .62594 & .25040 & .07401 & .0176 & 1.8 & -.54897 & .73326 & .73148 & .36137 & .12742 & .03562 \\
\hline 1.9 & -.48028 & .86242 & .68533 & .29538 & .09316 & .0235 & 1.9 & -.63314 & .66996 & .78045 & .41915 & .15836 & .04717 \\
\hline 2.0 & -.57436 & .82885 & .74128 & .34378 & .11546 & .0309 & 2.0 & -.69946 & .59149 & .82038 & .47901 & .19360 & .06130 \\
\hline 2.1 & -.65617 & .78173 & .79250 & .35913 & .14106 & .0399 & 2.1 & -.74627 & .49945 & .84967 & .53983 & .23310 & .07832 \\
\hline 2.2 & -.72413 & .72163 & .83774 & .44886 & .17007 & .0508 & 2. 2 & -.77241 & .39585 & .86697 & .60035 & .27668 & .09849 \\
\hline 2. 3 & -.77693 & .64940 & .87581 & .50430 & .20253 & .0638 & 2.3 & -.77730 & .28300 & 87113 & .65919 & .32406 & .12204 \\
\hline 2.4 & -.81358 & .56312 & .90559 & .56057 & .23843 & .0791 & 2.4 & -.76094 & .16347 & .86133 & .71492 & .37480 & .14916 \\
\hline 2.5 & -.83344 & .47313 & .92609 & .61713 & .27767 & .0969 & 2.5 & -.72387 & .04007 & .83704 & .76605 & .42832 & .17998 \\
\hline 2.6 & -.83620 & .37194 & .93645 & .67274 & .32008 & .1173 & 3.6 & -.66720 & -.08429 & .79809 & .81109 & .48391 & .21455 \\
\hline 2.7 & -.82187 & .26425 & .93599 & .72653 & .36541 & .1406 & 2. 7 & -.59251 & -.20662 & .74467 & .84860 & .54071 & .25282 \\
\hline 2.8 & -.79084 & .15191 & .92417 & .77748 & .41331 & .1667 & 2.8 & -.50188 & -.32395 & .67732 & .87720 & .59776 & .29468 \\
\hline 2.9 & -.74378 & .03689 & .90970 & .82455 & .46336 & .1959 & 2.9 & -.39778 & -.43342 & .59698 & .89565 & .65397 & .33987 \\
\hline 3.0 & -.68169 & -.07879 & .86544 & .86671 & .51504 & .2280 & 3.0 & -.28304 & -.53230 & .50491 & .90288 & .70819 & .38807 \\
\hline 3.1 & -.60588 & -.19306 & .81852 & .90294 & .56778 & .2632 & 3.1 & -.16073 & -.61815 & .40271 & .89799 & .75919 & .43880 \\
\hline 3.2 & -.51789 & -.30385 & .76025 & .93228 & .62090 & .3012 & 3.2 & -.03414 & -.68880 & .29229 & .88035 & .80570 & .49150 \\
\hline 3.3 & -.41952 & -.40913 & .69118 & .95383 & .67367 & .3421 & 3.3 & .09336 & -.74245 & .17581 & .84957 & .84646 & .54547 \\
\hline 3.4 & -.31275 & -.50398 & .61208 & .96678 & .72531 & .3855 & 3.4 & .21838 & -.77773 & .05562 & .80553 & .88022 & .59993 \\
\hline 3.5 & -.19973 & -.59557 & .52393 & .97043 & .77498 & .4312 & 3.5 & .33760 & -.79369 & -.06575 & .74846 & .90580 & .65400 \\
\hline 3.6 & -.08273 & -.67325 & .42789 & .96420 & .82181 & .4788 & 3.6 & .44787 & -.78990 & -.18570 & .67884 & .92210 & .70669 \\
\hline 3.7 & .03592 & -.73854 & .32530 & .94766 & .86491 & .5279 & 3.7 & .54627 & -.76640 & -.30157 & .59751 & .92815 & .75697 \\
\hline 3.8 & .15386 & -.79021 & .21766 & .92055 & .90341 & .5781 & 3.8 & .63021 & -.72371 & -.41077 & .50559 & .92316 & .80375 \\
\hline 3.9 & .26874 & -.82725 & .10660 & .88277 & .93643 & .6288 & 3.9 & .69748 & -.66289 & -.51081 & 40449 & .90649 & .84591 \\
\hline 4.0 & .37828 & -.84894 & -.00616 & .83440 & .96312 & .6793 & 4.0 & .74631 & -.58540 & -.59934 & .29588 & .87774 & .88235 \\
\hline 4.1 & .48031 & -.85483 & -.11881 & .77570 & .98271 & .7291 & 4.1 & .77541 & -.49317 & -.67427 & .18167 & 83673 & .91197 \\
\hline 4. 2 & .57280 & -.84477 & -.22953 & .70714 & .99446 & .7774 & 4. 2 & .78404 & -.38851 & -.73379 & .06396 & .78356 & .93373 \\
\hline 4.3 & .65394 & -.81891 & -.33647 & .62936 & .99773 & .8235 & 4.3 & -..... & -.27402 & -.77642 & -.05500 & .71856 & .94667 \\
\hline 4.4 & .72211 & -.77770 & -.43783 & .54318 & .99200 & .8665 & 4.4 & -..... & -.15260 & -.80107 & -.17286 & .64235 & .94995 \\
\hline 4.5 & .77597 & -.72188 & -.53186 & .44960 & .97684 & .9058 & 4.5 & -..... & -.02731 & -.80705 & -.28722 & .55581 & .94284 \\
\hline 4.6 & .81447 & -.65248 & -.61693 & .34977 & .95196 & .9406 & 4. 6 & ........ & .09867 & -.79413 & -.39570 & 46009 & .92479 \\
\hline 4.7 & .83684 & -.57077 & -.69152 & .24500 & ....... & .9700 & 4.7 & ........ & .22214 & -.76251 & -.49597 & .35657 & .89543 \\
\hline 4.8 & .84264 & -.47827 & -.75430 & .13672 & -...... & .9934 & 4. 8 & - n- & .33996 & -.71286 & -.58585 & .24689 & .85459 \\
\hline 4. 9 & .83177 & -.37673 & -.80411 & .02644 & -....... & 1. 0100 & 4.9 & - . & .44911 & -.64627 & -.66332 & .13283 & .80233 \\
\hline 5.0 & .80444 & -.26805 & -.84001 & -.08423 & ......... & 1. 0193 & 5.0 & ...... & .54679 & -.56425 & -.72661 & .01639 & .73891 \\
\hline & & & & & & & 5.1 & ........ & .63050 & -.46872 & -.77423 & -.10035 & .66487 \\
\hline
\end{tabular}


Table 2,-Continued

\begin{tabular}{|c|c|c|c|c|c|c|c|c|c|c|c|c|c|}
\hline \multicolumn{7}{|c|}{$\mathrm{E}_{\mathrm{lab} .}=150 \mathrm{Mev}$} & \multicolumn{7}{|c|}{$\mathrm{E}_{\mathrm{lab} \cdot}=150 \mathrm{Mev}$} \\
\hline$x$ & $u_{0}(x)$ & $u_{1}(x)$ & $u_{2}(x)$ & $u_{3}(x)$ & $u_{4}(x)$ & $u_{5}(x)$ & $x$ & $u_{0}(x)$ & $u_{1}(x)$ & $u_{2}(x)$ & $u_{3}(x)$ & $u_{4}(x)$ & $u_{5}(x)$ \\
\hline 0.1 & 0.22994 & 0.01427 & 0.00046 & & & & 2.6 & -0.40735 & -0.41883 & 0.55142 & 0.84835 & 0.62495 & 0.32625 \\
\hline .2 & .42044 & .05357 & .00350 & 0.00017 & 0.00001 & & 2.7 & -.28848 & -.52147 & .45161 & .85348 & .68178 & .37771 \\
\hline .3 & .54636 & .11272 & .01129 & .00081 & .00005 & & 2.8 & -.15996 & -.60809 & .34040 & .84405 & .73443 & .43202 \\
\hline .4 & .62237 & 18662 & .02555 & .00249 & .00019 & 0.00001 & 2. 9 & -.02609 & -.67594 & .22042 & .81932 & .78123 & .48839 \\
\hline .5 & .65321 & .27031 & .04749 & .00585 & .00057 & .00005 & 3.0 & .10865 & -.72286 & .09468 & .77893 & .82051 & .54584 \\
\hline .6 & .64279 & .35898 & .07786 & .01166 & .00136 & .00013 & 3.1 & .23977 & -.74733 & -.03359 & .72298 & .85065 & .60327 \\
\hline .7 & .59923 & .44801 & .11691 & .02072 & .00285 & .00032 & 3.2 & .36293 & -.74853 & -.16093 & .65201 & .87017 & .65942 \\
\hline .8 & .52488 & .53306 & .16443 & .03382 & .00537 & .00070 & 3.3 & .47405 & -.72634 & -.28387 & .56703 & .87776 & .71295 \\
\hline .9 & .42627 & .61016 & .21972 & .05169 & .00932 & .00137 & 3.4 & .56946 & -.68141 & -.39895 & .46949 & .87232 & .76242 \\
\hline 1.0 & .30905 & .67573 & .28166 & .07495 & .01517 & .00250 & 3.5 & .64601 & -.61509 & -.50288 & .36126 & .85304 & .80636 \\
\hline 1.1 & .17895 & .72670 & .34873 & .10406 & .02342 & .00428 & 3.6 & .70120 & -.52942 & -.59262 & .24462 & .81941 & .84331 \\
\hline 1.2 & .04166 & .76058 & .41908 & .13930 & .03461 & .00695 & 3.7 & .73320 & -.42707 & -.66549 & .12216 & .77130 & $\begin{array}{l}.87001 \\
.87182\end{array}$ \\
\hline 1.3 & -.09728 & .77548 & .49057 & .18071 & .04925 & .01080 & 3.8 & .74098 & -.31123 & -.71928 & -.00327 & .70894 & .89058 \\
\hline 1.4 & -.23255 & .77021 & .56086 & .22807 & .06785 & .01616 & 3.9 & .72430 & -.18554 & -.75230 & -.12860 & .63296 & .89836 \\
\hline 1.5 & -.35923 & .74425 & .62749 & .28090 & .09085 & .02340 & 4. 0 & .68372 & -.05397 & -.76344 & -.25069 & .54429 & .89416 \\
\hline 1. 6 & -.47285 & .69782 & .68796 & .33844 & .11860 & .03290 & 4. 1 & .62058 & .07930 & -.75225 & -.36638 & .44465 & .87717 \\
\hline 1. 7 & -.56952 & .63180 & .73984 & .39965 & .15133 & .04508 & 4.2 & .53697 & .21006 & -.71892 & -.47259 & .33553 & .84685 \\
\hline 1.8 & -.64604 & .54777 & .78082 & .46325 & .18915 & .06033 & 4.3 & .43567 & .33413 & -.66433 & -.56641 & & .80297 \\
\hline 1.9 & -.69991 & .44792 & .80884 & .52771 & .23199 & .07905 & 4.4 & .32001 & .44755 & -.59000 & -.64522 & & .74561 \\
\hline 2.0 & -.72948 & .33499 & .82217 & .59122 & .27958 & .10158 & 4.5 & .19381 & .54668 & -.49805 & -.70675 & & .67521 \\
\hline 2.1 & -.73390 & .21218 & .81945 & .65222 & .33147 & .12821 & 4. 6 & .06122 & .62834 & -.39117 & -.74917 & & .59254 \\
\hline 2.2 & -.71318 & .08307 & .79977 & .70846 & .38698 & .15915 & 4. 7 & -.07338 & .68990 & -.27251 & -.77114 & & .49873 \\
\hline 2.3 & -.66817 & -.04853 & .76273 & .75807 & .44526 & .19450 & 4.8 & -.20556 & .72937 & -.14562 & -.77190 & & .39525 \\
\hline 2.4 & -.60050 & -.17866 & .70846 & .79911 & .50521 & .23426 & 4. 9 & -.33098 & .74546 & -.01431 & -.75124 & & .28390 \\
\hline 2.5 & -.51255 & -.30337 & .63762 & .82975 & .56558 & .27828 & 5.0 & -.44551 & .73763 & .11743 & -.70960 & & .16675 \\
\hline
\end{tabular}

TABLE $3 . \quad \delta_{l}$ (radians)

\begin{tabular}{|c|c|c|c|c|c|}
\hline \multirow{2}{*}{$x$} & \multicolumn{5}{|c|}{$E_{\text {lab. }}(\mathrm{Mev})$} \\
\hline & 20 & 50 & 90 & 120 & 150 \\
\hline 0 & 1. 5886 & 1. 2958 & 1. 1255 & 1. 0483 & 0. 9907 \\
\hline 1 & $0.1871_{5}$ & 0. 3078 & 0.3607 & 0.3767 & .3844 \\
\hline 2 & .0314 & .0878 & .1331 & $.1566_{5}$ & .1723 \\
\hline 3 & .0040 & .0284 & .0555 & .0712 & .0839 \\
\hline 4 & .0007 & .0049 & $.0234_{5}$ & .0339 & .0424 \\
\hline 5 & $.0000_{5}$ & .0027 & $.0103_{5}$ & .0168 & .0223 \\
\hline
\end{tabular}

Los Angeles, February 12, 1953. 